\title{
Nutrient Status of Aprioct (Prunus armeniaca L.) Orchards of District Kargil, Ladakh, India
}

\author{
Mansoor Ali ${ }^{1 *}$, M.A. Malik ${ }^{2}$, Tsering Dolkar ${ }^{3}$ and Ahmad Ali ${ }^{1}$ \\ ${ }^{1}$ Division of Soil Science, ${ }^{2}$ Division of Fruit Science, Sher-e-Kashmir University of \\ Agricultural Science and Technology Kashmir, India \\ ${ }^{3}$ Collage of Agriculture Engineering, Sher-e-Kashmir University of Agricultural Science and \\ Technology Kashmir, India \\ *Corresponding author
}

\section{Keywords \\ Apricot, Soil, Orchards, District Kargil, available nutrients and micro nutrients}

\section{Article Info}

Accepted: 02 May 2018 Available Online: 10 June 2018

\section{A B S T R A C T}

Aprioct (Prunus armeniaca L.) is the main fruit in Ladakh region. Soil nutrients play a vital role in the growth, development and yield of apricot tree and the information on the nutritional status of an area can go a long way in planning judicious fertilizers and soil management practices to develop economically viable alternatives for the orchardists. Twenty one orchards with uniform age and vigour were selected and surveyed (simple random survey) for the purpose of collection of soil and leaf samples in District Kargil. The soil samples were collected from the three different depths viz., $0-20 \mathrm{~cm}, 20-40 \mathrm{~cm}$ and 40-60 cm. The soils were mostly sandy loam with sand, silt and clay with neutral to alkaline in reaction. Most of the soil samples were within the safe limit of salinity and were grouped under class $\mathrm{A}<0.5 \mathrm{dsm}^{-1}$. Organic carbon is almost low irrespective of all locations. While in available nutrients contents nitrogen is low at all locations, phosphorus is adequate and potassium is varied from medium to high category. The available calcium and magnesium did not reveal a definite pattern along the depth. The available sulphur content in these soils was sufficient and did not exhibit any definite trend in its vertical distribution. The DTPA-extractable zinc was found to be low, copper was found to be optimum in $95.23 \%$ soil samples. Iron was found low in $42.85 \%$ soil samples and medium to high in $57.14 \%$ soil samples. Manganese was found to be low in almost all samples with the surface soils containing higher amount than the sub-surface soils. All the micronutrients cations showed a decreasing trend with the increase in soil depth.

\section{Introduction}

Apricot (Prunus armeniaca L.) belongs to family Rosaceae. It is grown mostly in the temperate regions of the world e.g. Western and Central Asia, Europe, North Western
Himalaya and Western Tibet. It is probably a native of Western China. In India, it is cultivated in Jammu and Kashmir, Himachal Pradesh and hills of Uttar Pradesh. In Jammu and Kashmir State, about 3030 hectare area covered under this fruit. Ladakh is named as 
cold arid of North-Western Himalayas in Indian Subcontinent. Apricot being the most dominant fruit crop in the Ladakh region. The market value of the fruit (Apricot) produced from the cold arid areas, is almost double that of the fruit produced from other temperate and sub temperate parts of the country. Seedling originated apricots grow successfully up to an altitude of 3,500 meters a.m.s.l. Ladakh comprises of Kargil and Leh district of Jammu and Kashmir state and is spread over an geographical area of 96,701 $\mathrm{km}^{2}$ accounting for $43 \%$ of the area of the state and $75 \%$ of the cold arid region of the India. Fruit crops are raised in 47 villages of Kargil and 54 villages of Leh District, based on the length of the cropping season and elevation Sharma and Mir (1997) identified three climatic divisions (i.e., the upper, middle and lower) within the cold arid region.

The lower belt includes area from Saspol to Batalik, Nubra valley and larger parts of Kargil. Double cropping is practiced in these lower belts and in fruit production, both in terms of variety and quantity is maximum in these areas. The apricot growing areas in District Kargil are Karkitchoo, Hardass, Hunderman, Shilikchey, Poyen, Akchamal, Batalik, Chulichan, Gargardo, Darchiks, Garkhon and Sanjak. These apricot growing areas are situated between $34.5^{\circ}$ to $34.7^{\circ}$ North latitude, $76.2^{\circ}$ to $76.5^{\circ}$ East longitude and at an altitude of 2,500-2,750 meters a.m.s.1.

Generally speaking, the soils in Ladakh show a very wide variation in texture-sandy, loamy, silty loam, etc. and crops perform differently in the different soil types about which there is very little awareness among the local farmers (Singh, 1995). The sand content in the soils of Kargil (Kurbathang plateau) is relatively less (51.29 to $93.00 \%)$ with sandy loam as predominant surface texture. The soils in Drass areas are relatively finer with sandy loam to clay loam as the dominant texture while as the soils in the Leh (igoo-phey command area) are coarse textured with sand content of $95.0 \%$ and clay content ranging from 1.20 to $2.40 \%$. The percentage of sand in Thicksay is relatively less (50 to 60\%) with a clay content varying between 13-22\%. Soils are thus loose with good aeration and lack in the capacity to absorb and hold the sufficient moisture and nutrients (Talib 1986). $\mathrm{pH}$ and Electrical conductivity of District Kargil ranged between 7.5 to 8.5 in April and 8.0 to 8.7 in September for all locations. While-as in the sub-surface layer the value stood at $>8.0$, 0.002 to $0.080 \quad(\mathrm{mmhos} / \mathrm{cm})$ respectively reported in Apricot orchards of district Kargil (Norboo, 1994) and Sanjay et al., (2005) also reported electrical conductivity varied from 0.05 to 1.41 and 0.08 to $1.55 \mathrm{dS} \mathrm{m}^{-1}$ respectively in soils of Leh and Kargil District and further confirmed that electrical conductivity were within the safe limits (grouped under class $\mathrm{A},<0.5 \mathrm{dS} \mathrm{m}^{-1}$ ).

Whereas the organic carbon content were ranged between 0.7 to 14.1 and 1.4 to $35.8 \mathrm{~g}$ $\mathrm{kg}^{-1}$ respectively in both districts. Macronutrients like Nitrogen, Phosphorus and Potassium were varied from 91.99 to 223.66 $\mathrm{kg} / \mathrm{ha}$ and phosphorus is within the normal range irrespective of locations and various soil depths (Norboo, 1994). While as potassium varied from 11 to 496 and 103 to $861 \mathrm{~kg} / \mathrm{ha}$ in soil samples of Leh and Kargil District, respectively (Sanjay et al., (2005). While studying the micro-nutrients $(\mathrm{Zn}, \mathrm{Cu}, \mathrm{Fe}$ and $\mathrm{Mn})$ in the soils of Ladakh it has been reported that Zinc ( 0.08 to $2.08 \mathrm{mg} / \mathrm{kg}$ ), copper ( 0.28 to $1.24 \mathrm{mg} / \mathrm{kg}$ ), Fe (1.56 to $14.62 \mathrm{mg} / \mathrm{kg}), \mathrm{Mn}$ (2.12 to 5.92) (Jalali et al., 2000). Soil nutrients play a vital role in the growth, development and yield of plant and the information on the nutritional status of an area can go a long way in planning judicious fertilizers and soil management practices to develop economically viable alternatives for the farming community. 


\section{Materials and Methods}

\section{Survey of study area}

Investigations were carried out in 21 orchards of uniform age, vigour and growth selected from main apricot growing areas of District Kargil located at different locations and accordingly soil samples and leaf samples were collected under the standard procedure. A comprehensive survey was conducted in order to evaluate apricot growing orchards at different locations in District Kargil. The design of the survey used in achieving the objectives of the current study was simple random sampling. The Global Positioning System (GPS) co-ordinates (Table 1) were taken along the sampling sites and were used to draw the Arc Map (Map 1) in Arc GIS 10.2.

\section{Climate}

Cold arid zone is experienced by harsh climate dryness from extreme heat and cold, excessive dryness and very low rainfall and as such this region is called the cold arid or cold desert zone of the state. Based on the length of the cropping season and elevation Sharma and Mir (1997) identified three climatic divisions (i.e., the upper, middle and lower) within the cold arid region. The lower belt includes area from Saspol to Batalik, Nubra valley and larger parts of Kargil. Double cropping is practiced in this area, both in terms of variety and quantity is maximum in this area.

\section{Soil}

The soils are coarse in texture, shallow and sandy derived from weathered debris of rocks. Thee soils falls under the order "Entisols" and sub group Typic-cryoranthents (Gawande et al., 1979). The soils are coarse in texture, low in organic matter and high in calcium carbonate percentage (Takkar and Randhawa, 1978; Katyal and Aggarwal, 1982).

\section{Vegetation}

With the changing weather pattern, cropping pattern in the cold desert region is changing rapidly and farmers have shifted from old traditional crops like buckwheat, barley, black peas to low volume high value crops such as peas potato, apple, apricot, almond and seabuckthorn. In fodders alfa alfa is widely grown. Willows (Salix species) and Poplars (Populas species) are found along the Indus basin.

The soil samples were collected from the drip line of fruit trees from 21 different apricot orchards in different blocks of Kargil at different depths i.e., $0-20 \mathrm{~cm}, 20-40 \mathrm{~cm}$ and $40-60 \mathrm{~cm}$. The soil samples after collection were dried, crushed and sieved through $2 \mathrm{~mm}$ sieve and packed for analysis. Mechanical properties (Piper, 1966), $\mathrm{pH}$ and electrical conductivity (1:2.5 soil: water ratio), Organic carbon (Walkey and Black, 1934), nitrogen (Subbiah and Asija, 1956), phosphorus (Jackson, 1973). Potassium (Jackson, 1973), sulphur (Chesnin and Yien, 1951) exchangeable calcium and magnesium (Black 1965). The available (DTPA-extractable) micronutrients $(\mathrm{Zn}, \mathrm{Cu}, \mathrm{Fe}$ and $\mathrm{Mn})$ contents of the soil were determined by Lindsay and Norvell (1978). Simple coefficient of correlation ' $r$ ' was worked out between various soil properties as per the standard procedures given by Gomez and Gomez (1984). All the data was analyzed and computed with the help of statistical tool SPSS 16.0. Confidence interval (C.I) was also worked out by using the same statistical tool.

\section{Results and Discussion}

Soil separates in profile soil samples showed a varied distribution in apricot orchards of District Kargil. The content of sand, silt and clay varied from 55.64 to $60.43,24.05$ to 27.06 and 14.84 to $17.43 \%$ respectively with a 
mean value of 58.12, 25.66 and $16.12 \%$ respectively in surface soils and in sub-surface soils with mean value of $61.40,23.35$ and $15.08 \%$ respectively at $95 \%$ confidence interval. (C.I) (Table 2). The majority of the soil samples were having sandy loam texture which is in line with the findings of (Norboo, 1994; Jalali, 2000; Sanjay et al., 2005) and geographically the study area comes under the Cold arid region where the soils are mostly sandy in texture.

$\mathrm{pH}$ value in surface soils ranged from 7.72 to 8.10 while as in sub-surface layers it varied from 7.78 to 8.15 . The $\mathrm{pH}$ value obtained at different soil depths showed an increasing trend with the increase in the soil depth exhibiting significant differences among locations (Table 3), similar results were reported by Minhas and Singh (1980), Norboo (1994) and Akhtar (2005). Electrical conductivity varied from 0.50 to $0.68 \mathrm{dsm}^{-1}$ with a mean value of $0.59 \mathrm{dsm}^{-1}$ in surface soils at $95 \%$ confidence interval. While as in sub-surface layers, it varied from 0.36 to 0.52 with mean a value of $0.44 \mathrm{dsm}^{-1}$ in surface soils at $95 \%$ confidence interval (C.I) and it doesn't show a definite pattern along with the depth of the soil (Table 3).

These results are in conformity with Norboo (1994), Sanjay et al., (2005) and Colak et al., (2010). Organic carbon present in the soil samples varied from 0.15 to $0.20 \%$ with a mean value of $0.18 \%$ significantly at $95 \%$ Confidence interval in surface layer while-as in sub-surface layers it varied from 0.11 to $0.16 \%$ with a mean value of $0.13 \%$ at $95 \%$ C.I. (Table 3). Similar reports were reported in their findings by Norboo (1994), Sanjay et al., (2005) and Colak et al., (2010). Calcium carbonate content varied from 0.28 to $0.56 \%$ with a mean value of $0.45 \%$ while-as in the sub-surface layers it varied from 0.16 to $0.75 \%$ with a mean value of $0.51 \%$ statistically at $95 \%$ C.I. (Table 3). The available nutrients Nitrogen, Phosphorus and Potassium in the surface soils were varied from 225.28 to $253.79 \mathrm{~kg} / \mathrm{ha}, 21.67$ to 26.57 $\mathrm{kg} / \mathrm{ha}, 244.35$ to $322.32 \mathrm{~kg} / \mathrm{ha}$ respectively in the surface soils at $95 \%$ C.I. while as in the sub surface soils it varied from 158.57 to $192.42 \mathrm{~kg} / \mathrm{ha}, 20.32$ to $24.69 \mathrm{~kg} / \mathrm{ha}, 156.79$ to $231.30 \mathrm{~kg} / \mathrm{ha}$ respectively statistically at $95 \%$ C.I. (Table 4).

Available nitrogen may be in low category in soils of apricot orchards of Kargil because of the fact that mineralization of organic matter is very slow in cold arid regions due to low temperature (Sharma and Triparthi, 2002) and loss of nitrogen through leaching under light textured soils (Folh and Turk, 1973) where as results are in accordance with the findings of Norboo (1994) and Sanjay et al., (2005) for available phosphorus and potassium. The Calcium, Magnesium and Sulphur were varied from 218.72 to $236.17 \mathrm{ppm}, 15.17$ to 21.82 $\mathrm{ppm}, 11.26$ to $11.66 \mathrm{~kg} / \mathrm{ha}$ respectively in the upper $15 \mathrm{~cm}$ or surface soils while as in sub surface it had mean value of 210.29 ppm, $18.77 \mathrm{ppm}$ and $11.39 \mathrm{~kg} / \mathrm{ha}$ respectively at 95\% C.I. (Table 4).

This is in conformity with the findings of Bhandari and Randhawa (1985), Norboo (1994), Dar (1996) and Najar (2002). The data revealed that available sulphur did not exhibited any definite trend in its vertical distribution (Table 4) these results are in agreement with those of Arora et al., (1989), Sharma and Bhandari (1992) and Wani (2001).

DTPA-extractable micro nutrients $(\mathrm{Zn}, \mathrm{Cu}, \mathrm{Fe}$ and $\mathrm{Mn}$ ) content in the apricot orchard soils of District kargil varied from 0.53 to $0.59 \mathrm{mg} / \mathrm{kg}$, 0.79 to $1.29 \mathrm{mg} / \mathrm{kg}, 4.76$ to $6.97 \mathrm{mg} / \mathrm{kg}$ and 1.30 to $2.21 \mathrm{mg} / \mathrm{kg}$ respectively in the surface soils. In sub surface soils it varied from 0.57 to $0.70 \mathrm{mg} / \mathrm{kg}, 0.54$ to $0.85 \mathrm{mg} / \mathrm{kg}, 3.41$ to $5.81 \mathrm{mg} / \mathrm{kg}$ and 0.79 to $1.21 \mathrm{mg} / \mathrm{kg}$ 
respectively at $95 \%$ C.I. (Table 5). The results were supported by the findings of Jalali et al., (2000) and Sanjay et al., (2005). The amount of available zinc, copper, iron and manganese in the soils under study decreased with an increase in soil depth with maximum amount in surface soils, which can be attributed to the accumulation of high organic matter in surface soils. Similar reports were reported by Sharma et al., (2004), Sharma and Chaudhary (2007) and Dar et al., (2011). The overall manganese content in all samples was below the critical limits as prescribed by Nayyer et al., (1985).

Perusal of the correlation coefficient values between physico-chemical properties with available nutrients in the surface soils revealed that $\mathrm{pH}$ of soils had significant negative correlation with phosphorus $(\mathrm{r}=-0.566)$, sulphur $(\mathrm{r}=-0.508)$ and iron $(\mathrm{r}=-0.484)$ (Table 6). A similar relationship between $\mathrm{pH}$ and available phosphorus has been reported by the Zaho et al., (2011) and Khokhar et al., (2012). Significant and negative relationship of $\mathrm{pH}$ with available sulphur has also been reported by Cheema and Arora (1984), Arora et al., (1989), Pandey et al., (1989) and Dar et al., (2011).

Similar relationship between the $\mathrm{pH}$ and available iron has been reported by Chibba and Sekhon (1985), Jalali et al., (1989), Ramesh et al., (1994), Gupta and Srivastava (1990), Mir (1994), Zahoor et al., (2005), Sharma et al., (2005) and Dar et al., (2011). Electrical conductivity showed a significantly negative correlation with nitrogen $(\mathrm{r}=-0.534)$ (Table 6). Khokhar et al., (2012) also reported the same.

Soil organic carbon showed positive and significant correlation with nitrogen $(\mathrm{r}=$ $0.498)$ phosphorus $(\mathrm{r}=0.508)$, potassium $(\mathrm{r}=$ $0.424)$, sulphur $(\mathrm{r}=0.469)$ zinc $(\mathrm{r}=0.473)$, copper $(\mathrm{r}=0.482)$, iron $(\mathrm{r}=0.497)$ and manganese $(r=0.432)$ in surface soils (Table 6). Similar results have been reported by
Singh and Ahuja (1990), Mushki (1994), Wani (2001), Akhtar (2005) and Najar et al., (2006). Panday et al., (2000), Wani (2001) and Akhtar (2005). The relationship between organic carbon and available potassium is in conformity with the findings of Chibba and Sekhon (1985), Kumar et al., (1987), Mandal et al., (1990), Mongia and Bandyopadhyay (1991) and Wani (2001). Calcium carbonate revealed a positive and significant correlation with calcium $(r=421)$, magnesium (428) and Clay $(\mathrm{r}=471)$ (Table 6).

The relationship of calcium carbonate with available calcium and magnesium observed was also supported by the findings of Jassal et al., (2000), Wani (2001) and Dar et al., (2011). The clay content showed significant and positive correlation with nitrogen $(r=$ 0.475) but showed negative but nonsignificant correlation with potassium ( $\mathrm{r}=-$ $0.254)$, calcium $(\mathrm{r}=-0.131)$, sulphur $(\mathrm{r}=-$ $0.164)$, manganese $(\mathrm{r}=-0.103)$, zinc $(\mathrm{r}=-$ 0.117 ) (Table 6). The results were in line with Mandal et al., (1990), Gupta et al., (1980) and Akhtar (2005).

In sub surface soils $\mathrm{pH}$ had significant and negative correlation with phosphorus ( $\mathrm{r}=-$ $0.552)$, iron $(\mathrm{r}=-0.620)$. Electrical conductivity revealed a negative and significant relationship with available nitrogen ( $\mathrm{r}=-$ 0.498). Organic carbon content revealed positive and significant correlation with nitrogen $(0.542)$, phosphorus $(\mathrm{r}=0.501)$, potassium $(\mathrm{r}=0.525)$, sulphur $(\mathrm{r}=0.491)$ and zinc $(r=0.531)$, copper $(r=0.513)$, iron $(r=$ $0.493)$ and manganese $(r=0.471)$. A negative and significant correlation of calcium carbonate with nitrogen $(\mathrm{r}=-0.481)$ and phosphorus $(\mathrm{r}=-0.537)$. The clay content of sub-surface soils revealed significant and positive correlation with available nitrogen ( $\mathrm{r}$ $=0.493$ ), while-as, it showed non-significant correlation with all other nutrients under study. 
Table.1 Soil and plant sampling sites (District Kargil)

\begin{tabular}{|c|c|l|c|c|c|}
\hline $\begin{array}{c}\text { Soil } \\
\text { sample }\end{array}$ & $\begin{array}{c}\text { Plant } \\
\text { sample }\end{array}$ & $\begin{array}{c}\text { Location } \\
\text { Latitude } \\
\text { (North- } \\
\text { south) }\end{array}$ & $\begin{array}{c}\text { Longitude } \\
\text { (East-West) }\end{array}$ & $\begin{array}{c}\text { Altitude (meter, } \\
\text { amsl) }\end{array}$ \\
\hline S1 & P1 & Karkichu & 34.63 & 76.25 & 2840 \\
\hline S2 & P2 & Hardas & 34.61 & 76.25 & 2665 \\
\hline S3 & P3 & Hunderman & 34.61 & 76.24 & 2661 \\
\hline S4 & P4 & Shiliksay & 34.82 & 76.09 & 2680 \\
\hline S5 & P5 & Akchmal & 34.59 & 76.23 & 2871 \\
\hline S6 & P6 & Poyen & 34.74 & 76.08 & 2710 \\
\hline S7 & P7 & Gongma Kargil & 34.79 & 76.23 & 2920 \\
\hline S8 & P8 & Chulichan & 34.85 & 76.53 & 2700 \\
\hline S9 & P9 & Batalik & 34.76 & 76.40 & 2803 \\
\hline S10 & P10 & Darchik & 34.63 & 76.15 & 2675 \\
\hline S11 & P11 & Gargardo & 34.84 & 76.46 & 2695 \\
\hline S12 & P12 & Garkhon & 34.63 & 76.62 & 2724 \\
\hline S13 & P13 & Sanjak & 34.53 & 76.69 & 2784 \\
\hline S14 & P14 & Menji & 34.57 & 76.00 & 2778 \\
\hline S15 & P15 & Titichumik & 34.51 & 76.13 & 2780 \\
\hline S16 & P16 & Hotriculture orchard & 34.70 & 76.22 & 2816 \\
\hline S17 & P17 & kurbathang orchard & 34.70 & 76.16 & 2824 \\
\hline S18 & P18 & KVK Orchard & 34.71 & 76.18 & 2816 \\
\hline S19 & P19 & MARES Kargil & 34.71 & 76.22 & 2874 \\
\hline S20 & P20 & Aba Grong & 34.59 & 76.23 & 2868 \\
\hline S21 & P21 & Pishu & & & \\
\hline & & & & \\
\hline
\end{tabular}

Table.2 Mechanical properties of soils of apricot orchards of District Kargil

\begin{tabular}{|c|c|c|c|c|c|}
\hline Sites & Depth (cm) & Sand (\%) & Silt (\%) & Clay (\%) & Textural class \\
\hline \multirow[t]{3}{*}{ Karkichu } & $0-20$ & 55.80 & 29.32 & 14.88 & Sandy loam \\
\hline & $20-40$ & 59.25 & 27.29 & 13.46 & Sandy loam \\
\hline & $40-60$ & 60.75 & 25.37 & 13.88 & Sandy loam \\
\hline \multirow[t]{3}{*}{$\overline{\text { Hardas }}$} & $0-20$ & 55.29 & 29.36 & 15.35 & Sandy loam \\
\hline & $20-40$ & 57.39 & 28.45 & 14.16 & Sandy loam \\
\hline & $40-60$ & 59.29 & 27.37 & 13.34 & Sandy loam \\
\hline \multirow[t]{3}{*}{ Hunderman } & $0-20$ & 64.03 & 20.05 & 15.92 & Sandy loam \\
\hline & $20-40$ & 66.37 & 18.36 & 15.27 & Sandy loam \\
\hline & $40-60$ & 69.47 & 17.43 & 13.10 & Sandy loam \\
\hline \multirow[t]{3}{*}{ Shiliksay } & $0-20$ & 56.16 & 25.03 & 18.81 & Sandy loam \\
\hline & $20-40$ & 58.92 & 24.02 & 17.06 & Sandy loam \\
\hline & $40-60$ & 59.47 & 23.45 & 17.08 & Sandy loam \\
\hline \multirow[t]{3}{*}{ Akchmal } & $0-20$ & 54.37 & 29.67 & 15.96 & Sandy loam \\
\hline & $20-40$ & 58.45 & 28.36 & 13.19 & Sandy loam \\
\hline & $40-60$ & 59.95 & 27.45 & 12.60 & Sandy loam \\
\hline \multirow[t]{3}{*}{ Poyen } & $0-20$ & 54.45 & 28.45 & 17.10 & Sandy loam \\
\hline & $20-40$ & 55.35 & 27.05 & 17.60 & Sandy loam \\
\hline & $40-60$ & 57.17 & 25.35 & 17.48 & Sandy loam \\
\hline \multirow[t]{3}{*}{ Gongma Kargil } & $0-20$ & 61.37 & 28.37 & 10.26 & Sandy loam \\
\hline & $20-40$ & 62.04 & 25.39 & 12.57 & Sandy loam \\
\hline & $40-60$ & 63.95 & 24.71 & 11.34 & Sandy loam \\
\hline
\end{tabular}


Contd...

\begin{tabular}{|c|c|c|c|c|c|}
\hline Sites & Depth (cm) & Sand (\%) & Silt (\%) & Clay (\%) & Textural class \\
\hline \multirow[t]{3}{*}{ Chulichan } & $0-20$ & 59.45 & 28.95 & 11.60 & Sandy loam \\
\hline & $20-40$ & 62.42 & 26.45 & 11.13 & Sandy loam \\
\hline & $40-60$ & 63.92 & 24.36 & 11.72 & Sandy loam \\
\hline \multirow[t]{3}{*}{ Batalik } & $0-20$ & 65.41 & 19.27 & 15.32 & Sandy loam \\
\hline & $20-40$ & 67.02 & 18.42 & 14.56 & Sandy loam \\
\hline & $40-60$ & 68.92 & 17.56 & 13.52 & Sandy loam \\
\hline \multirow[t]{3}{*}{ Darchik } & $0-20$ & 54.08 & 28.42 & 17.50 & Sandy loam \\
\hline & $20-40$ & 58.25 & 26.16 & 15.59 & Sandy loam \\
\hline & $40-60$ & 69.49 & 24.21 & 6.30 & Sandy loam \\
\hline \multirow[t]{3}{*}{ Gargardo } & $0-20$ & 56.31 & 28.21 & 15.48 & Sandy loam \\
\hline & $20-40$ & 57.21 & 26.12 & 16.67 & Sandy loam \\
\hline & $40-60$ & 60.32 & 25.01 & 14.67 & Sandy loam \\
\hline \multirow[t]{3}{*}{ Garkhon } & $0-20$ & 63.42 & 21.01 & 15.57 & Sandy loam \\
\hline & $20-40$ & 64.92 & 19.02 & 16.06 & Sandy loam \\
\hline & $40-60$ & 66.42 & 17.95 & 15.63 & Sandy loam \\
\hline \multirow[t]{3}{*}{ Sanjak } & $0-20$ & 57.32 & 26.32 & 16.36 & Sandy loam \\
\hline & $20-40$ & 59.72 & 25.11 & 15.17 & Sandy loam \\
\hline & $40-60$ & 62.31 & 23.56 & 14.13 & Sandy loam \\
\hline \multirow[t]{3}{*}{ Menji } & $0-20$ & 57.76 & 26.31 & 15.93 & Sandy loam \\
\hline & $20-40$ & 59.42 & 24.67 & 15.91 & Sandy loam \\
\hline & $40-60$ & 63.23 & 23.01 & 13.76 & Sandy loam \\
\hline
\end{tabular}

Contd...

\begin{tabular}{|c|c|c|c|c|c|}
\hline Sites & Depth (cm) & Sand $(\%)$ & Silt (\%) & Clay $(\%)$ & Textural class \\
\hline \multirow[t]{3}{*}{ Titichumik } & $0-20$ & 65.11 & 19.42 & 15.47 & Sandy loam \\
\hline & $20-40$ & 67.23 & 17.33 & 15.44 & Sandy loam \\
\hline & $40-60$ & 69.41 & 16.23 & 14.36 & Sandy loam \\
\hline \multirow{3}{*}{$\begin{array}{l}\text { Horticulture } \\
\text { orchard }\end{array}$} & $0-20$ & 52.31 & 28.13 & 19.56 & Sandy loam \\
\hline & $20-40$ & 54.42 & 27.04 & 18.54 & Sandy loam \\
\hline & $40-60$ & 56.33 & 25.45 & 18.22 & Sandy loam \\
\hline \multirow{3}{*}{$\begin{array}{l}\text { kurbathang } \\
\text { orchard }\end{array}$} & $0-20$ & 64.32 & 23.42 & 12.26 & Sandy loam \\
\hline & $20-40$ & 66.42 & 22.22 & 11.36 & Sandy loam \\
\hline & $40-60$ & 69.81 & 21.32 & 8.87 & Sandy loam \\
\hline \multirow[t]{3}{*}{ Kvk Orchard } & $0-20$ & 51.42 & 29.12 & 19.46 & Sandy loam \\
\hline & $20-40$ & 53.92 & 27.11 & 18.97 & Sandy loam \\
\hline & $40-60$ & 55.22 & 25.27 & 19.51 & Sandy loam \\
\hline \multirow[t]{3}{*}{ MARES, Kargil } & $0-20$ & 50.13 & 28.14 & 21.73 & Sandy loam \\
\hline & $20-40$ & 52.75 & 26.24 & 21.01 & Sandy loam \\
\hline & $40-60$ & 54.12 & 25.27 & 20.61 & Sandy loam \\
\hline \multirow[t]{3}{*}{ Aba Grong } & $0-20$ & 67.95 & 18.21 & 13.84 & Sandy loam \\
\hline & $20-40$ & 68.08 & 16.31 & 15.61 & Sandy loam \\
\hline & $40-60$ & 69.02 & 14.21 & 16.77 & Sandy loam \\
\hline \multirow[t]{3}{*}{ Pishu } & $0-20$ & 52.32 & 27.18 & 20.50 & Sandy loam \\
\hline & $20-40$ & 54.52 & 25.12 & 20.36 & Sandy loam \\
\hline & $40-60$ & 56.32 & 24.02 & 19.66 & Sandy loam \\
\hline \multicolumn{2}{|c|}{ Mean (Surface) } & 58.12 & 25.66 & 16.12 & \\
\hline \multicolumn{2}{|c|}{$95 \%$ C.I } & $55.64-60.43$ & 24.05-27.60 & $14.84-17.43$ & \\
\hline \multirow{2}{*}{\multicolumn{2}{|c|}{ Mean (Sub-surface) }} & 61.40 & 23.35 & 15.08 & \\
\hline & $95 \%$ C.I & 59.14-63.67 & 21.71-25.19 & 13.81-16.49 & \\
\hline
\end{tabular}


Table.3 Physico-chemical properties of soils of apricot orchards of District Kargil

\begin{tabular}{|c|c|c|c|c|c|}
\hline Location site & $\begin{array}{c}\text { Depth } \\
\text { (cm) }\end{array}$ & pH (1:2.5) & $\mathrm{EC}\left(\mathrm{dsm}^{-1}\right)$ & $\mathrm{OC}(\%)$ & $\mathrm{CaCO}_{3}(\%)$ \\
\hline \multirow{3}{*}{ Karkichu } & $0-20$ & 8.25 & 0.34 & 0.10 & 0.31 \\
\hline & $20-40$ & 8.34 & 0.28 & 0.08 & 0.25 \\
\hline & $40-60$ & 8.67 & 0.25 & 0.08 & 0.26 \\
\hline \multirow[t]{3}{*}{ Hardas } & $0-20$ & 7.60 & 0.76 & 0.23 & 0.25 \\
\hline & $20-40$ & 7.07 & 0.28 & 0.08 & 0.21 \\
\hline & $40-60$ & 7.68 & 0.33 & 0.10 & 0.22 \\
\hline \multirow[t]{3}{*}{ Hunderman } & $0-20$ & 7.65 & 0.25 & 0.08 & 0.31 \\
\hline & $20-40$ & 7.68 & 0.34 & 0.10 & 0.24 \\
\hline & $40-60$ & 7.71 & 0.39 & 0.12 & 0.26 \\
\hline \multirow[t]{3}{*}{ Shiliksay } & $0-20$ & 8.20 & 0.34 & 0.10 & 0.31 \\
\hline & $20-40$ & 8.24 & 0.37 & 0.11 & 0.42 \\
\hline & $40-60$ & 8.42 & 0.41 & 0.12 & 0.42 \\
\hline \multirow[t]{3}{*}{ Akchmal } & $0-20$ & 8.48 & 0.64 & 0.19 & 0.33 \\
\hline & $20-40$ & 8.50 & 0.59 & 0.18 & 0.34 \\
\hline & $40-60$ & 8.53 & 0.52 & 0.16 & 0.33 \\
\hline \multirow[t]{3}{*}{ Poyen } & $0-20$ & 8.45 & 0.54 & 0.16 & 0.34 \\
\hline & $20-40$ & 8.29 & 0.57 & 0.17 & 0.26 \\
\hline & $40-60$ & 8.24 & 0.65 & 0.20 & 0.25 \\
\hline \multirow{3}{*}{$\begin{array}{l}\text { Gongma } \\
\text { Kargil }\end{array}$} & $0-20$ & 7.92 & 0.98 & 0.29 & 0.34 \\
\hline & $20-40$ & 8.01 & 0.88 & 0.26 & 0.29 \\
\hline & $40-60$ & 8.04 & 0.62 & 0.19 & 0.28 \\
\hline \multirow[t]{3}{*}{ Chulichan } & $0-20$ & 7.25 & 0.69 & 0.21 & 0.25 \\
\hline & $20-40$ & 7.32 & 0.24 & 0.07 & 0.24 \\
\hline & $40-60$ & 7.29 & 0.25 & 0.08 & 0.25 \\
\hline \multirow[t]{3}{*}{ Batalik } & $0-20$ & 8.29 & 0.48 & 0.14 & 0.26 \\
\hline & $20-40$ & 8.56 & 0.34 & 0.10 & 0.23 \\
\hline & $40-60$ & 8.40 & 0.37 & 0.11 & 0.21 \\
\hline \multirow[t]{3}{*}{ Darchik } & $0-20$ & 7.39 & 0.42 & 0.13 & 0.37 \\
\hline & $20-40$ & 7.23 & 0.33 & 0.10 & 0.34 \\
\hline & $40-60$ & 8.02 & 0.11 & 0.03 & 0.34 \\
\hline \multirow[t]{3}{*}{ Gargardo } & $0-20$ & 7.14 & 0.58 & 0.17 & 0.27 \\
\hline & $20-40$ & 7.31 & 0.31 & 0.09 & 0.25 \\
\hline & $40-60$ & 7.29 & 0.26 & 0.08 & 0.27 \\
\hline
\end{tabular}


Contd...

\begin{tabular}{|c|c|c|c|c|c|}
\hline Location site & $\begin{array}{l}\text { Depth } \\
\text { (cm) }\end{array}$ & pH $(1: 2.5)$ & $\mathrm{EC}\left(\mathrm{dsm}^{-1}\right)$ & $\mathrm{OC}(\%)$ & $\mathrm{CaCO}_{3}(\%)$ \\
\hline \multirow[t]{3}{*}{ Garkhon } & $0-20$ & 8.45 & 0.48 & 0.14 & 0.26 \\
\hline & $20-40$ & 8.42 & 0.43 & 0.13 & 0.24 \\
\hline & $40-60$ & 8.40 & 0.41 & 0.12 & 0.21 \\
\hline \multirow[t]{3}{*}{ Sanjak } & $0-20$ & 8.35 & 0.62 & 0.19 & 0.31 \\
\hline & $20-40$ & 8.34 & 0.49 & 0.15 & 0.28 \\
\hline & $40-60$ & 8.55 & 0.50 & 0.15 & 0.29 \\
\hline \multirow[t]{3}{*}{ Menji } & $0-20$ & 7.68 & 0.72 & 0.22 & 0.27 \\
\hline & $20-40$ & 7.52 & 0.35 & 0.11 & 0.24 \\
\hline & $40-60$ & 7.54 & 0.62 & 0.19 & 0.25 \\
\hline \multirow[t]{3}{*}{ Titichumik } & $0-20$ & 7.91 & 0.51 & 0.15 & 0.21 \\
\hline & $20-40$ & 7.93 & 0.45 & 0.14 & 0.15 \\
\hline & $40-60$ & 7.95 & 0.16 & 0.05 & 0.11 \\
\hline \multirow{3}{*}{$\begin{array}{l}\text { Horticulture } \\
\text { orchard }\end{array}$} & $0-20$ & 7.97 & 0.96 & 0.29 & 1.41 \\
\hline & $20-40$ & 7.81 & 0.82 & 0.25 & 3.11 \\
\hline & $40-60$ & 7.76 & 0.76 & 0.23 & 3.26 \\
\hline \multirow{3}{*}{$\begin{array}{l}\text { Kurbathang } \\
\text { orchard }\end{array}$} & $0-20$ & 7.52 & 0.64 & 0.19 & 0.41 \\
\hline & $20-40$ & 7.60 & 0.29 & 0.09 & 0.36 \\
\hline & $40-60$ & 8.42 & 0.40 & 0.12 & 0.32 \\
\hline \multirow{3}{*}{$\begin{array}{l}\text { KVK } \\
\text { Orchard }\end{array}$} & $0-20$ & 7.53 & 0.82 & 0.25 & 1.21 \\
\hline & $20-40$ & 7.62 & 0.76 & 0.23 & 0.75 \\
\hline & $40-60$ & 7.64 & 0.74 & 0.22 & 0.72 \\
\hline \multirow{3}{*}{$\begin{array}{l}\text { MARES } \\
\text { Kargil }\end{array}$} & $0-20$ & 7.78 & 0.65 & 0.20 & 0.65 \\
\hline & $20-40$ & 7.80 & 0.59 & 0.18 & 0.61 \\
\hline & $40-60$ & 7.79 & 0.57 & 0.17 & 0.58 \\
\hline \multirow[t]{3}{*}{ Aba Grong } & $0-20$ & 8.20 & 0.54 & 0.16 & 0.45 \\
\hline & $20-40$ & 8.30 & 0.46 & 0.14 & 0.37 \\
\hline & $40-60$ & 8.31 & 0.42 & 0.13 & 0.38 \\
\hline \multirow[t]{3}{*}{ Pishu } & $0-20$ & 8.10 & 0.46 & 0.14 & 0.31 \\
\hline & $20-40$ & 7.96 & 0.32 & 0.10 & 0.32 \\
\hline & $40-60$ & 7.84 & 0.29 & 0.09 & 0.28 \\
\hline \multicolumn{2}{|c|}{ Mean (Surface) } & 7.90 & 0.59 & 0.18 & 0.45 \\
\hline \multicolumn{2}{|c|}{$95 \%$ C.I } & 7.72-8.10 & $0.50-0.68$ & $0.15-0.20$ & $0.28-0.56$ \\
\hline \multicolumn{2}{|c|}{ Mean (Sub-surface) } & 7.96 & 0.44 & 0.13 & 0.51 \\
\hline \multicolumn{2}{|c|}{$95 \%$ C.I } & 7.78-8.15 & $0.36-0.52$ & 0.11-0.16 & $0.16-0.75$ \\
\hline
\end{tabular}


Table.4 Available Macronutrients in soil samples of apricot orchards of District Kargil

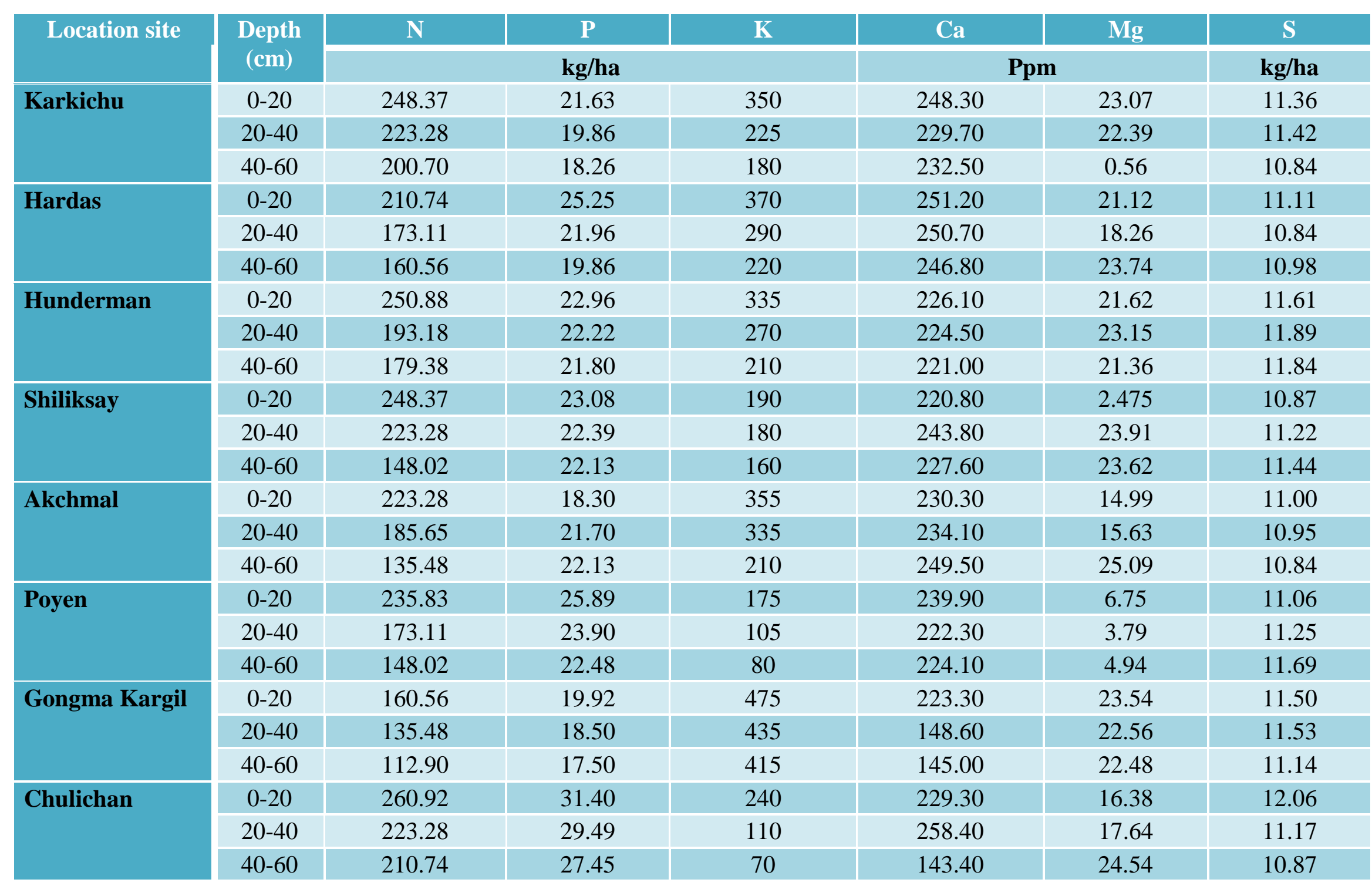


Contd...

\begin{tabular}{|c|c|c|c|c|c|c|c|}
\hline \multirow[t]{2}{*}{ Location site } & $\begin{array}{c}\text { Depth } \\
\text { (cm) }\end{array}$ & $\mathbf{N}$ & $\mathbf{P}$ & $\mathbf{K}$ & $\mathrm{Ca}$ & Mg & $\mathbf{S}$ \\
\hline & & \multicolumn{3}{|c|}{$\mathrm{kg} / \mathrm{ha}$} & \multicolumn{2}{|c|}{ Ppm } & kg/ha \\
\hline \multirow[t]{3}{*}{ Batalik } & $0-20$ & 273.46 & 18.44 & 240 & 226.60 & 21.99 & 11.14 \\
\hline & $20-40$ & 223.28 & 16.49 & 175 & 242.30 & 13.16 & 11.47 \\
\hline & $40-60$ & 135.48 & 16.28 & 145 & 253.50 & 1.88 & 11.17 \\
\hline \multirow[t]{3}{*}{ Darchik } & $0-20$ & 223.28 & 32.57 & 195 & 220.30 & 14.60 & 11.00 \\
\hline & $20-40$ & 185.65 & 30.83 & 145 & 196.60 & 20.40 & 10.76 \\
\hline & $40-60$ & 148.02 & 29.80 & 110 & 219.60 & 23.97 & 10.60 \\
\hline \multirow[t]{3}{*}{ Gargardo } & $0-20$ & 260.92 & 34.55 & 260 & 221.56 & 16.46 & 12.12 \\
\hline & $20-40$ & 229.56 & 32.42 & 230 & 220.12 & 17.36 & 12.46 \\
\hline & $40-60$ & 210.74 & 31.57 & 210 & 184.30 & 23.45 & 12.49 \\
\hline \multirow[t]{3}{*}{ Garkhon } & $0-20$ & 273.46 & 28.10 & 335 & 230.50 & 23.66 & 10.89 \\
\hline & $20-40$ & 260.92 & 26.92 & 175 & 91.05 & 18.17 & 11.25 \\
\hline & $40-60$ & 223.28 & 26.18 & 140 & 99.05 & 23.42 & 11.89 \\
\hline \multirow[t]{3}{*}{ Sanjak } & $0-20$ & 273.46 & 17.50 & 130 & 167.00 & 24.72 & 12.00 \\
\hline & $20-40$ & 235.83 & 16.66 & 70 & 158.60 & 22.41 & 11.31 \\
\hline & $40-60$ & 210.74 & 15.51 & 60 & 238.30 & 20.76 & 11.28 \\
\hline \multirow[t]{3}{*}{ Menji } & $0-20$ & 260.92 & 30.44 & 250 & 198.40 & 16.74 & 11.31 \\
\hline & $20-40$ & 173.11 & 28.89 & 165 & 256.10 & 22.04 & 11.17 \\
\hline & $40-60$ & 110.39 & 26.73 & 110 & 241.60 & 12.13 & 11.31 \\
\hline \multirow[t]{3}{*}{ Titichumik } & $0-20$ & 185.65 & 18.41 & 160 & 246.40 & 16.86 & 11.39 \\
\hline & $20-40$ & 85.30 & 19.68 & 115 & 250.80 & 24.06 & 11.03 \\
\hline & $40-60$ & 122.93 & 20.30 & 105 & 156.10 & 23.60 & 10.87 \\
\hline
\end{tabular}


Contd...

\begin{tabular}{|c|c|c|c|c|c|c|c|}
\hline \multirow[t]{2}{*}{ Location site } & $\begin{array}{l}\text { Depth } \\
(\mathrm{cm})\end{array}$ & $\mathbf{N}$ & $\mathbf{P}$ & $\mathbf{K}$ & $\mathrm{Ca}$ & $\mathrm{Mg}$ & $\mathbf{S}$ \\
\hline & & \multicolumn{3}{|c|}{ kg/ha } & \multicolumn{2}{|c|}{ Ppm } & kg/ha \\
\hline \multirow{3}{*}{$\begin{array}{l}\text { Horticulture } \\
\text { orchard }\end{array}$} & $0-20$ & 260.92 & 31.79 & 360 & 236.40 & 25.00 & 11.55 \\
\hline & $20-40$ & 198.20 & 30.07 & 215 & 274.10 & 22.19 & 11.31 \\
\hline & $40-60$ & 160.56 & 29.53 & 175 & 243.10 & 0.03 & 11.03 \\
\hline \multirow{3}{*}{$\begin{array}{l}\text { Kurbathang } \\
\text { orchard }\end{array}$} & $0-20$ & 210.74 & 23.56 & 365 & 236.60 & 0.93 & 11.72 \\
\hline & $20-40$ & 173.11 & 22.13 & 190 & 223.30 & 13.38 & 11.44 \\
\hline & $40-60$ & 97.84 & 21.35 & 150 & 156.10 & 24.08 & 11.14 \\
\hline \multirow[t]{3}{*}{ KKk Orchard } & $0-20$ & 260.92 & 20.12 & 320 & 221.36 & 25.36 & 12.43 \\
\hline & $20-40$ & 210.74 & 18.75 & 280 & 222.15 & 22.17 & 12.20 \\
\hline & $40-60$ & 135.48 & 18.06 & 240 & 210.45 & 21.86 & 12.00 \\
\hline \multirow[t]{3}{*}{ MARES, Kargil } & $0-20$ & 248.37 & 23.19 & 305 & 210.35 & 24.16 & 11.53 \\
\hline & $20-40$ & 185.65 & 21.59 & 270 & 164.36 & 23.56 & 11.42 \\
\hline & $40-60$ & 148.02 & 20.54 & 245 & 156.32 & 22.15 & 11.36 \\
\hline \multirow[t]{3}{*}{ Aba Grong } & $0-20$ & 198.20 & 19.08 & 255 & 245.60 & 23.45 & 11.86 \\
\hline & $20-40$ & 148.02 & 18.35 & 230 & 231.60 & 21.56 & 12.18 \\
\hline & $40-60$ & 97.84 & 17.50 & 215 & 231.80 & 22.01 & 12.00 \\
\hline \multirow[t]{3}{*}{ Pishu } & $0-20$ & 260.92 & 20.29 & 285 & 246.10 & 24.48 & 11.14 \\
\hline & $20-40$ & 235.83 & 18.81 & 255 & 236.12 & 23.84 & 11.47 \\
\hline & $40-60$ & 198.20 & 18.50 & 235 & 228.16 & 23.54 & 11.64 \\
\hline \multicolumn{2}{|c|}{ Mean (surface) } & 237.57 & 24.28 & 285.00 & 217.13 & 18.03 & 11.48 \\
\hline \multicolumn{2}{|c|}{ 95\% C.I } & 225.28-253.79 & 21.67-26.57 & 244.35-322.32 & 218.72-236.17 & 15.17-21.82 & 11.26-11.66 \\
\hline \multicolumn{2}{|c|}{ Mean (sub-surface) } & 175.39 & 22.57 & 196.48 & 210.29 & 18.77 & 11.39 \\
\hline \multicolumn{2}{|c|}{$95 \%$ C.I } & 158.57-192.42 & 20.32-24.69 & 156.79-231.30 & 193.79-229.42 & 16.53-21.61 & 11.19-11.59 \\
\hline
\end{tabular}


Table.5 Micro-nutrient status in soils of apricot orchards of District Kargil (mg/kg)

\begin{tabular}{|c|c|c|c|c|c|}
\hline Location site & $\operatorname{Depth}(\mathrm{cm})$ & Zn & $\mathrm{Cu}$ & $\mathrm{Fe}$ & Mn \\
\hline \multirow[t]{3}{*}{ Karkichu } & $0-20$ & 0.61 & 0.57 & 2.82 & 1.19 \\
\hline & $20-40$ & 0.34 & 0.55 & 2.32 & 0.99 \\
\hline & $40-60$ & 0.32 & 0.44 & 2.23 & 0.83 \\
\hline \multirow[t]{3}{*}{ Hardas } & $0-20$ & 0.54 & 2.78 & 11.99 & 1.23 \\
\hline & $20-40$ & 0.43 & 2.06 & 10.46 & 0.92 \\
\hline & $40-60$ & 0.41 & 1.24 & 5.69 & 0.39 \\
\hline \multirow[t]{3}{*}{ Hunderman } & $0-20$ & 0.58 & 1.15 & 4.36 & 2.14 \\
\hline & $20-40$ & 0.57 & 0.81 & 4.01 & 1.78 \\
\hline & $40-60$ & 0.41 & 0.74 & 3.98 & 0.86 \\
\hline \multirow[t]{3}{*}{ Shiliksay } & $0-20$ & 0.57 & 1.53 & 4.35 & 2.51 \\
\hline & $20-40$ & 0.56 & 1.10 & 3.90 & 1.34 \\
\hline & $40-60$ & 0.46 & 0.96 & 2.68 & 0.55 \\
\hline \multirow{3}{*}{ Akchmal } & $0-20$ & 0.65 & 1.39 & 4.39 & 1.69 \\
\hline & $20-40$ & 0.54 & 0.98 & 2.19 & 1.49 \\
\hline & $40-60$ & 0.52 & 0.92 & 1.51 & 1.36 \\
\hline \multirow[t]{3}{*}{ Poyen } & $0-20$ & 0.55 & 0.57 & 7.22 & 1.41 \\
\hline & $20-40$ & 0.54 & 0.42 & 5.51 & 0.48 \\
\hline & $40-60$ & 0.46 & 0.30 & 3.54 & 0.39 \\
\hline \multirow{3}{*}{ Gongma Kargil } & $0-20$ & 0.71 & 0.34 & 4.21 & 1.03 \\
\hline & $20-40$ & 0.49 & 0.24 & 3.26 & 0.79 \\
\hline & $40-60$ & 0.43 & 0.23 & 3.20 & 0.64 \\
\hline \multirow[t]{3}{*}{ Chulichan } & $0-20$ & 0.57 & 1.20 & 7.67 & 1.49 \\
\hline & $20-40$ & 0.56 & 0.77 & 7.09 & 1.38 \\
\hline & $40-60$ & 0.55 & 0.69 & 6.41 & 0.50 \\
\hline \multirow{3}{*}{ Batalik } & $0-20$ & 0.58 & 0.71 & 4.35 & 2.22 \\
\hline & $20-40$ & 0.57 & 0.53 & 3.31 & 1.41 \\
\hline & $40-60$ & 0.56 & 0.44 & 2.73 & 1.25 \\
\hline \multirow[t]{3}{*}{ Darchik } & $0-20$ & 0.50 & 0.55 & 10.12 & 2.22 \\
\hline & $20-40$ & 0.31 & 0.47 & 14.37 & 1.41 \\
\hline & $40-60$ & 0.29 & 0.20 & 12.84 & 1.37 \\
\hline \multirow[t]{3}{*}{ Gargardo } & $0-20$ & 0.51 & 1.24 & 7.25 & 1.56 \\
\hline & $20-40$ & 0.48 & 0.30 & 6.68 & 1.31 \\
\hline & $40-60$ & 0.39 & 0.22 & 6.02 & 1.01 \\
\hline
\end{tabular}


Contd...

\begin{tabular}{|c|c|c|c|c|c|}
\hline Location site & $\operatorname{Depth}(\mathrm{cm})$ & Zn & $\mathrm{Cu}$ & $\mathrm{Fe}$ & Mn \\
\hline \multirow[t]{3}{*}{ Garkhon } & $0-20$ & 0.50 & 0.38 & 5.02 & 5.45 \\
\hline & $20-40$ & 0.33 & 0.30 & 2.41 & 2.86 \\
\hline & $40-60$ & 0.31 & 0.22 & 1.83 & 2.07 \\
\hline \multirow[t]{3}{*}{ Sanjak } & $0-20$ & 0.54 & 1.22 & 5.78 & 1.12 \\
\hline & $20-40$ & 0.50 & 0.77 & 5.47 & 1.03 \\
\hline & $40-60$ & 0.49 & 0.53 & 4.80 & 0.35 \\
\hline \multirow[t]{3}{*}{ Menji } & $0-20$ & 0.51 & 1.16 & 9.79 & 1.10 \\
\hline & $20-40$ & 0.40 & 0.98 & 7.58 & 0.79 \\
\hline & $40-60$ & 0.36 & 0.90 & 4.62 & 0.63 \\
\hline \multirow[t]{3}{*}{ Titichumik } & $0-20$ & 0.48 & 1.51 & 5.47 & 1.85 \\
\hline & $20-40$ & 0.40 & 1.41 & 4.89 & 0.94 \\
\hline & $40-60$ & 0.37 & 0.63 & 3.18 & 0.75 \\
\hline \multirow{3}{*}{$\begin{array}{l}\text { Horticulture } \\
\text { orchard }\end{array}$} & $0-20$ & 0.69 & 0.79 & 6.37 & 0.94 \\
\hline & $20-40$ & 0.46 & 0.77 & 4.75 & 0.37 \\
\hline & $40-60$ & 0.39 & 0.71 & 4.39 & 0.26 \\
\hline \multirow{3}{*}{$\begin{array}{l}\text { Kurbathang } \\
\text { orchard }\end{array}$} & $0-20$ & 0.56 & 1.02 & 4.17 & 2.68 \\
\hline & $20-40$ & 0.49 & 0.83 & 3.85 & 1.98 \\
\hline & $40-60$ & 0.48 & 0.81 & 1.96 & 0.57 \\
\hline \multirow[t]{3}{*}{ KVK Orchard } & $0-20$ & 0.64 & 1.56 & 6.48 & 1.56 \\
\hline & $20-40$ & 0.41 & 1.02 & 5.64 & 1.04 \\
\hline & $40-60$ & 0.36 & 0.94 & 5.03 & 0.86 \\
\hline \multirow[t]{3}{*}{ MARES, Kargil } & $0-20$ & 0.53 & 0.64 & 3.56 & 1.06 \\
\hline & $20-40$ & 0.50 & 0.58 & 3.38 & 0.92 \\
\hline & $40-60$ & 0.48 & 0.56 & 2.86 & 0.61 \\
\hline \multirow[t]{3}{*}{ Aba Grong } & $0-20$ & 0.53 & 0.81 & 4.58 & 1.21 \\
\hline & $20-40$ & 0.50 & 0.78 & 3.87 & 0.86 \\
\hline & $40-60$ & 0.48 & 0.73 & 3.16 & 0.61 \\
\hline \multirow[t]{3}{*}{ Pishu } & $0-20$ & 0.55 & 0.71 & 3.24 & 1.16 \\
\hline & $20-40$ & 0.43 & 0.64 & 3.14 & 1.06 \\
\hline & $40-60$ & 0.31 & 0.61 & 2.89 & 0.96 \\
\hline \multicolumn{2}{|c|}{ Mean (surface) } & 0.56 & 1.09 & 4.41 & 1.88 \\
\hline \multicolumn{2}{|c|}{$95 \%$ C.I } & 0.53-0.59 & $0.79-1.29$ & 4.76-6.97 & $1.30-2.21$ \\
\hline \multicolumn{2}{|c|}{ Mean (Sub-surface) } & 0.63 & 0.72 & 4.76 & 1.02 \\
\hline \multicolumn{2}{|c|}{ 95\% C.I } & $0.57-0.70$ & $0.54-0.85$ & $3.41-5.81$ & 0.79-1.21 \\
\hline
\end{tabular}


Table.6 Relationship between the physico-chemical characteristics and available soil nutrients in surface soils of apricot orchard of District Kargil

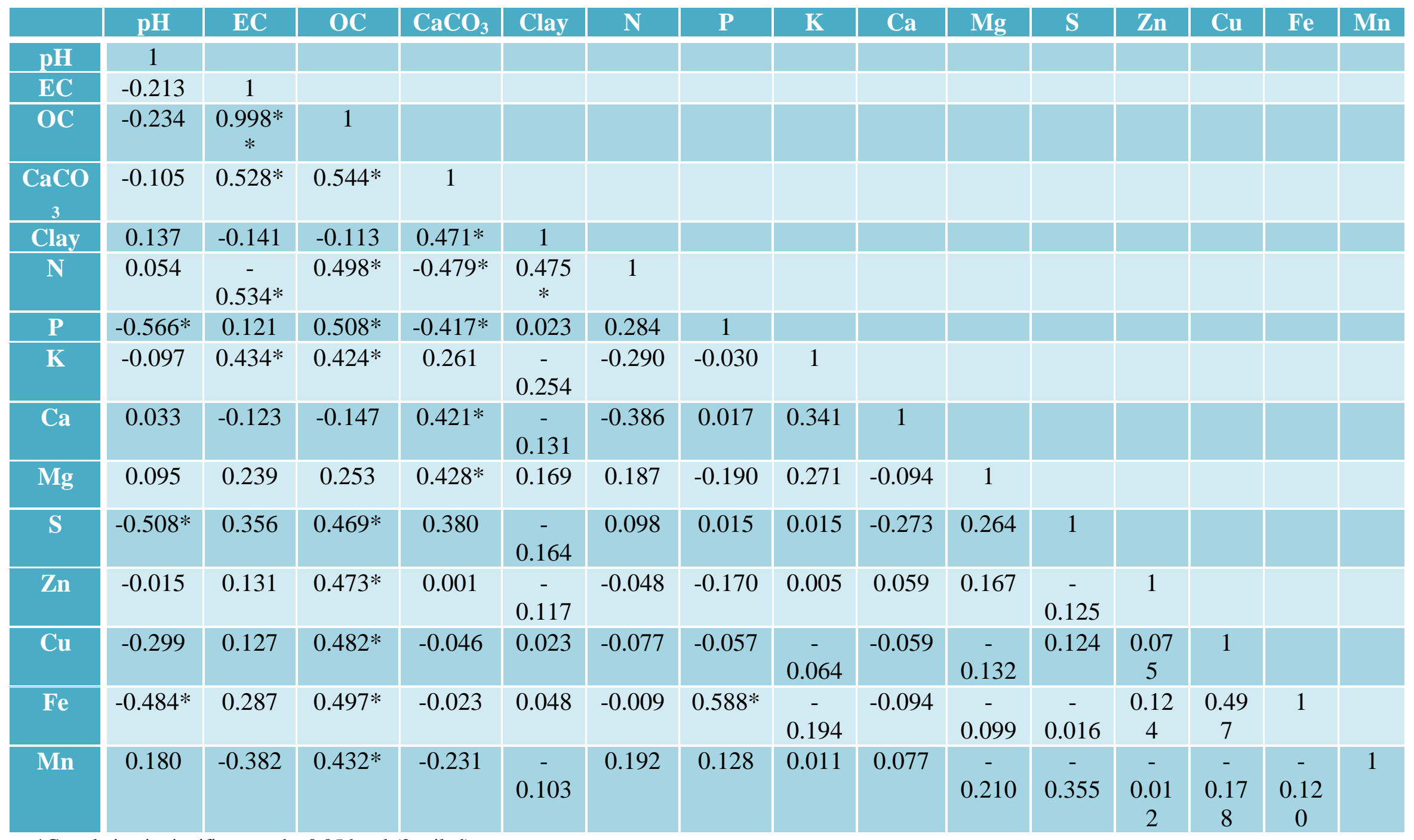

*Correlation is significant at the 0.05 level (2-tailed).

**Correlation is significant at the 0.01 level (2-tailed). 
Table.7 Relationship between the physico-chemical characteristics and available soil nutrients in sub-surface soils of apricot orchard of District Kargil

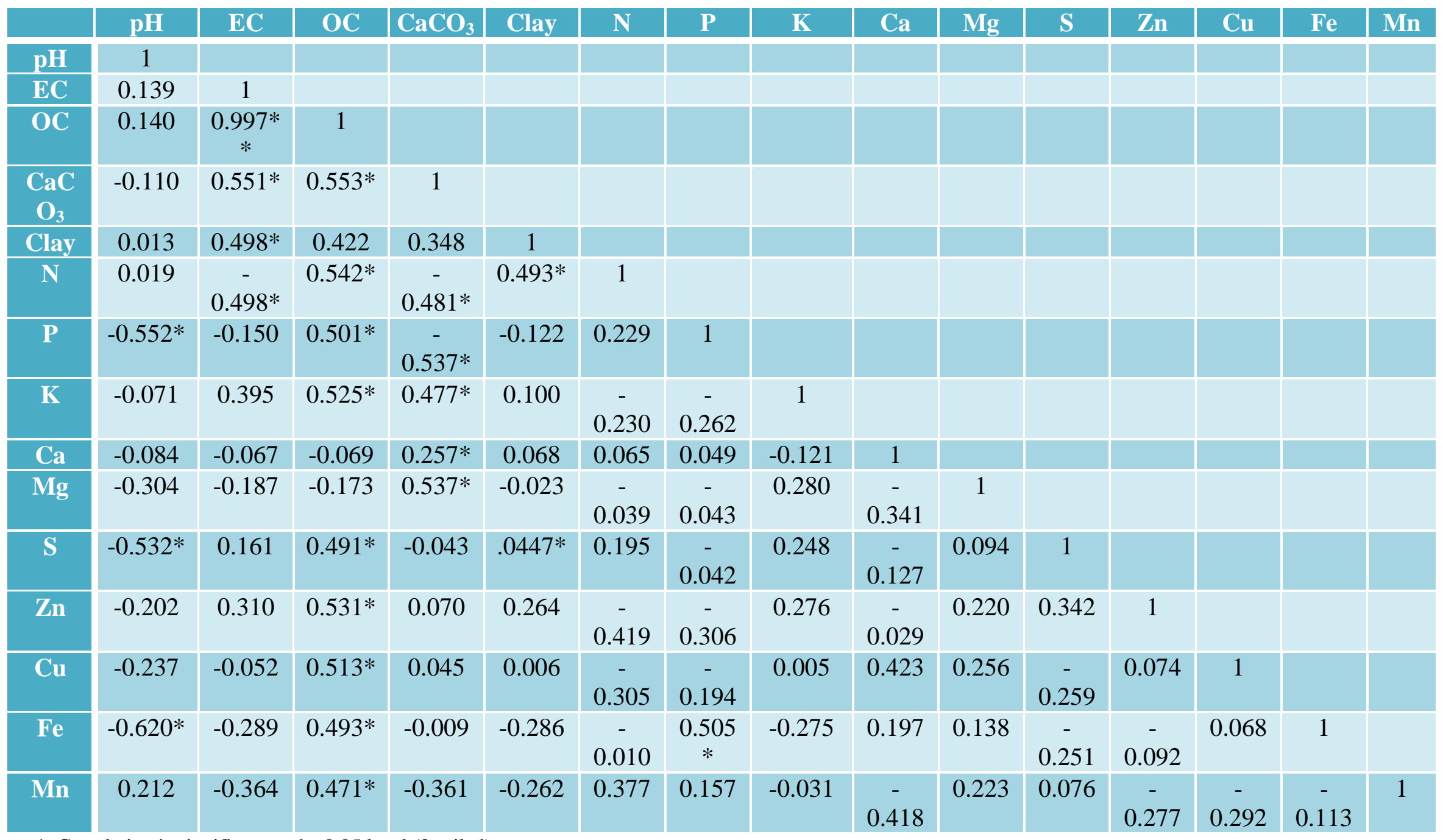

*. Correlation is significant at the 0.05 level (2-tailed).

**. Correlation is significant at the 0.01 level (2-tailed). 
Table.8 Nutrient status of apricot orchard of District Kargil (\%)

\begin{tabular}{|l|c|c|c|}
\hline \multicolumn{1}{|c|}{ Nutrients } & Low & Medium & High \\
\hline Nitrogen (N) & 100 & - & - \\
\hline Phosphorus (P) & - & 61.90 & 38.09 \\
\hline Potassium (K) & & 47.61 & 52.38 \\
\hline Zinc (Zn) & 76.19 & 23.80 & \\
\hline Copper (Cu) & - & 95.23 & \\
\hline Iron (Fe) & 42.85 & & 5.76 \\
\hline Manganese (Mn) & 100 & & \\
\hline
\end{tabular}

Table.9 Critical limits of available macro-nutrients in soil (Tandon, 1989)

\begin{tabular}{|l|c|c|c|}
\hline Nutrients & Low & Normal & High \\
\hline $\mathbf{N}$ & $<280 \mathrm{~kg} / \mathrm{ha}$ & $280-560 \mathrm{~kg} / \mathrm{ha}$ & $>560 \mathrm{~kg} / \mathrm{ha}$ \\
\hline $\mathbf{P}$ & $<10 \mathrm{~kg} / \mathrm{ha}$ & $10-25 \mathrm{~kg} / \mathrm{ha}$ & $>25 \mathrm{~kg} / \mathrm{ha}$ \\
\hline $\mathrm{K}$ & $<110 \mathrm{~kg} / \mathrm{ha}$ & $110-280 \mathrm{~kg} / \mathrm{ha}$ & $>280 \mathrm{~kg} / \mathrm{ha}$ \\
\hline O.C & $<0.50 \%$ & $0.50-0.75 \%$ & $>0.75 \%$ \\
\hline
\end{tabular}

Table.10 Critical limits of available micro-nutrients in soil

\begin{tabular}{|l|c|c|c|l|}
\hline \multicolumn{1}{|c|}{ Nutrients } & Low & Normal & High & \multicolumn{1}{c|}{ References } \\
\hline $\mathrm{Zn}(\mathrm{mg} / \mathrm{kg})$ & $<0.6$ & $0.6-1.2$ & $>1.2$ & Takkar and Mann $(1975)$ \\
\hline $\mathrm{Cu}(\mathrm{mg} / \mathrm{kg})$ & $<0.2$ & $0.2-2.0$ & $>2.0$ & Follet and Lindsay(1970) \\
\hline $\mathrm{Fe}(\mathrm{mg} / \mathrm{kg})$ & $<4.5$ & - & - & Follet and Lindsay(1970) \\
\hline $\mathrm{Mn}(\mathrm{mg} / \mathrm{kg})$ & $<3.74$ & - & - & Nayyar et al., (1985) \\
\hline
\end{tabular}

Map.1 GIS Arc map of sampling sites (District Kargil)

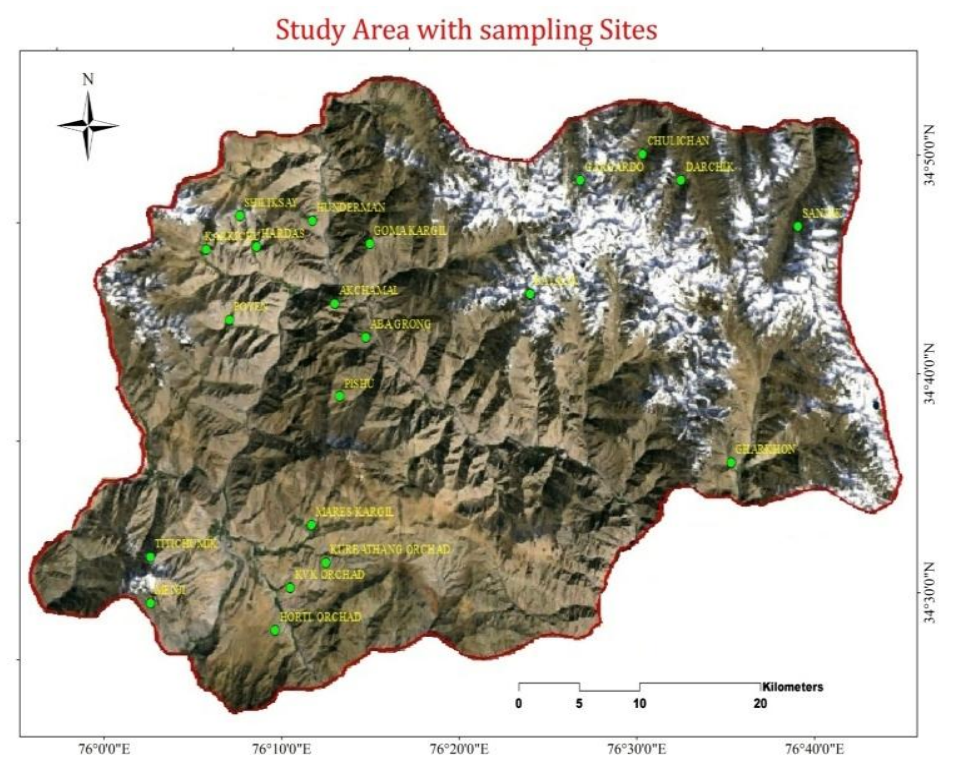


The overall soil nutrient status of apricot orchards of District Kargil was summarized in Table 8 based on different categorization limits prescribed by different researchers (Follet and Lindsay, 1970; Takkar and Mann, 1975; Nayyar et al., 1985 and Tandon, 1989) (Table 9 and 10). The overall nitrogen content in apricot orchards of District Kargil falls under low category while in case of phosphorus content $61.90 \%$ of soil samples fall under the medium category and $38.09 \%$ of the soil samples fall under the high category. In respect of potassium content in orchard soils $47.61 \%$ of soil samples falls under medium category and $52.38 \%$ falls under the high category. While observing the micronutrient status of apricot orchards of District Kargil available zinc content ranged from low category to medium category i.e., $76.19 \%$ soil samples were categorized under low category while $23.80 \%$ of soil samples fall under medium category. Similarly in case of copper content in the orchard soils of District Kargil $95.23 \%$ soil samples were categorized into medium category and $4.76 \%$ soil samples were categorized into the high status. While in case of iron content $42.85 \%$ soil samples falls under the low category and $57.14 \%$ soil samples were under medium to high category. Manganese content in all orchard soils falls within the low category.

From the summarized result of the present investigation it can be concluded that soils of apricot orchards of district Kargil were low in organic carbon and available nitrogen content but by and large adequately supplied with phosphorus, potassium, calcium, magnesium and sulhpur. Micronutrients like zinc, and manganese were low in these orchard soils. Iron content in these soils was low to adequate while as copper content of these orchard soils was sufficient. Poor nitrogen status of the orchard soils was matter of concern but it does not necessarily mean additional requirements as such until optimum values are determined with a deeper understanding of overall status for more seasons. Awareness campaigns should be conducted along with trainings by trained professionals to the farmers for better management of orchards. There is need to sort out the huge variations between soil and leaf analysis by way of standardizing the methods of nutrient extraction besides calculating the critical limits befitting environmental conditions of cold arid zones. Further and time to time surveys should be carried out in order to assess the nutrient status of soil, plant and its impact on quality of fruit. Microbiological parameters are also needed to study for the deeper understanding of microbial interactions in soil.

\section{References}

Akhtar, F. 2005. Studies on relationship between fruit yield and quality with soil and leaf nutrient content in apple orchards of Zangier block of district Baramulla Kashmir. Ph. D. Thesis submitted to Sher-e-Kashmir University of Agricultural Sciences \& Technology of Kashmir, Shalimar, Sriangar pp. 117.

Arora, B. R., Aulakh, M. S., Ghai, V. K. and Rosha, N. S. 1989. Profile distribution of sulphur in major soils of Punjab. Journal of Research, Punjab Agricultural University 26(2): 206-213.

Bhandari, A. R. and Randhawa, N. S. 1985. Distribution of available micro-nutrients in soils of apple orchards in Himachal Pradesh. Journal of the Indian Society of Soil Science 33: 171-174.

Black, C. A. 1965. Method of Soil Analysis, Part 2, Chemical and Microbiological Properties. American Society of Agronomy, Inc, Publisher, Madison, Wisconsin USA.

Cheema, H. S. and Arora, C. L. 1984. Sulphur status of soils, tube well waters and plants in some areas of Ludhiana 
underground wheat cropping system. Fertilizer News 29: 28-31.

Chesnin, L. and Yien, C. H. 1951. Turbidmetric determination of available sulphur. Proceeding of Soil Science Society of America 15: 149-151.

Chibba, I. M. and Sekhon, G. S. 1985. Effect of $\mathrm{pH}$ and organic carbon on availability of nutrients in acid soils. Journal of the Indian Society of Soil Science 36: 409411.

Colak, S., Celik, B., Gokalp, K., Yilmaz, K. U., Zengin, Y. and Paydas, K. S. 2010. Soil Nutritional Status of Apricot (Prunus armeniaca L.) Orchards in Malatya Province. Acta Horticulturae 862: 351-354.

Dar, M. A, Wani, J. A and Bhat, M. Y. 2011. Effect of physic-chemical characteristics on nutrient availability in cherry orchard soils of Kashmir. Journal of Hill Agriculture 2(2): 193196.

Dar, M. A. 1996. Nutrient status of cherry (Prunus avium) orchards of Srinagar district. M. Sc. (Agriculture) Thesis, submitted to Sher-e- Kashmir University of Agricultural Science and Technology of Kashmir. India pp. 140.

Folh, D. D. and Turk, L. M. 1973. Composition, manufacture and use of fertilizers. In: Fundamentals of Soil Science. Wiley Eastern Private Limited. New Delhi pp. 300-302.

Gawande, S. P., Sharma, S. P. and Chaman, R. K. 1979. Soils of camand area of abichenmethang canal in Ladakh and their management. Journal of Indian Society of Soil Science 27: 427-433.

Gomez, K. A. and Gomez, A. A. 1984. Statistical procedure for agriculture research. An International Rice Research Institute Book. $2^{\text {nd }}$ Edn. Willy Interscience publication, New York pp. 112.

Gupta, P. K. and Srivastava, P. C. 1990.
Distribution of different forms of micronutrient cations in some cultivated soils of Sikkim. Journal of Indian Society of Soil Science 38: 558-560.

Gupta, R.D., Jha, K.K. and Soni, B.P.1980. Studies on physical chemical and mineralogical nature of soils of Jammu and Kashmir. Current Agriculture 4: 34.

Jackson, M. L. 1973. Soil chemical analysis : Advanced course $\left(2^{\text {nd }}\right.$ Ed.). Madison, Wisconsin, USA.

Jalali, V. K., Gupta, J. P. and Razdan, H. K. 2000. Distribution of micronutrient cations in soils of Cold arid zone of Ladakh. Indian Journal of Agricultural Science 70: 128-130.

Jassal, H. S., Sidhu, P. S., Sharma, P. D and Mukhopadhyay. 2000. Mineralogy and geochemistry of some soils of Shiwalik hills. Journal of the Indian Society of Soil Science 48: 163-172.

Katyal, J. C. and Agarwala, S. C. 1982. Micronutrients research in India. Fertilizer News 27(2): 66-86.

Khokhar, Y., Harinder, S. R., Wasakha S. D., Gobinder, S. and Parkash S. G. 2012. Soil fertility and nutritional status of Kinnow orchards grown in aridisol of Punjab, India. African Journal of Agricultural Research 7(33): 46924697.

Kumar, R., Mahendra, S., Rahul, D. S. and Rajendra, S. 1987. Forms of potassium in some soils of Central Haryana. Journal of Research 4: 356-363.

Lindsay, W. L. and Norvell, W. A. 1978. Development of a DTPA soil test for zinc, iron, manganese and copper. Soil Science Society of American Journal 42: 421-428.

Mandal, A. K., Nath, S., Gupta, S. K. and Banerjee, S. K. 1990. Characterization and nutritional status of soils of middle and upper hill forest of the eastern Himalayas. Journal of the Indian 
Society of Soil Science 38: 100-106.

Minhas, R. S. and Singh, K. 1980. Soils of semi-arid highland region of India "A review". Agriculture Research 1(1): 1926.

Mongia, A. D. and Bandyopadhyay, A. K. 1991. Forms of potassium in the acidic hill soils of Andamans. Journal of the Indian Society of Soil Science 39: 573575.

Mushki, G. M. 1994. Studies on apple orchard soils of Kashmir M. Sc. (Agriculture) thesis, submitted to Sher-e-Kashmir University of Agriculture Science and Technology of Kashmir, India pp. 144.

Najar, G. R. 2002. Studies on pedogenesis and nutrient indexing of apple (Red Delicious) growing soils of the Kashmir. Ph. D (Agriculture) thesis, submitted to Sher-e-Kashmir University of Agriculture Science and Technology of Kashmir, India pp. 204.

Najar, G. R., Rahman, F. H. and Farida, A. 2006. Effect of altitude on distribution and forms of nitrogen in some apple growing soils of Kashmir. Environment and Ecology 24(2): 433-436.

Nayyar, V. K., Sandana, K. S. and Takkar, P. N. 1985. Methods and rates of $\mathrm{Mn}$ and its critical levels for wheat following rice on coarse textural soils. Fertilizer News 8: 173-78.

Norboo, S. 1994. Nutritional status of apricot plantation in some orchard areas of Kargil district. M. Sc. thesis submitted to Sher-e-Kashmir University of Agricultural Sciences \& Technology, J\&K, Srinagar pp. 125.

Panday, S. P., Singh, R. S. and Mishra, S. K. 2000. Availability of phosphorus and sulphur in inceptisols of Central Uttar Pradesh. Journal of the Indian Society of Soil Science 48(1): 118-121.

Pandey, D. K., Tiwari, K. N. and Tiwari, R. C. 1989. Different forms of sulphur in alluvial soils. Journal of Indian Society of Soil Science 37: 161-163.

Piper, C. S. 1966. Soil and Plant Analysis. Hans Publisher, Bombay, India.

Ramesh, R., Rao, H. P., Pillali, R. N., Reddy, T. and Rao, D. A. 1994. Correlation between soil chemical properties and available soil nutrients in relation to their fertility status. Journal of Indian Society of Soil Science 42: 322-323.

Sanjay, K., Dwivedi, Sharma, V. K. and Vipin, B. 2005. Status of Available Nutrients in Soils of Cold Arid Region of Ladakh. Journal of the Indian Society of Soil Science 53(3): 421-423.

Sharma, B. D., Harsh, A., Kumar, R. and Nayyar, V. K. 2004. Relationship between soil characteristics and total and DTPA- extractable micronutrients in inceptisols of Punjab. Communication in Soil Science and Plant Analysis 35(5-6): 799-818.

Sharma, J. C. and Chaudhary, S. K. 2007. Vertical distribution of micronutrient cations in relation to soil characteristics in lower Shiwaliks of Solan district of North-West Himalayas. Journal of the Indian Society of Soil science 55(1): 4044.

Sharma, J. P. and Mir. A. A. 1997. Crop improvement prospective in cold arid zones Ladakh, SKUAST, Regional Research Station, Leh Ladakh pp. 1-79.

Sharma, J. P. and Mir. A. A. 1997. Crop improvement prospective in cold arid zones Ladakh, SKUAST, Regional Research Station, Leh Ladakh pp. 1-79.

Sharma, U. and Bhandari, A. R. 1992. Survey of the nutrient status of apple orchards in Himachal Pradesh. Indian Journal of Horticulture 49(3): 234-241.

Sharma, U. and Tripathi, D. 2002. Status of available nutrients in soils of dry temperate zone of Himachal Pradesh. Annals of Plant and Soil Research 4(1): 167-170.

Singh, B. 1995. Vegetables in Ladakh. Field 
research laboratory (DRDO). C/O 56 APO.

Singh, K. and Ahuja, R. L. 1990. Distribution of primary nutrients in relation to soil characteristics in the Ghaggar river basin. Journal of the Indian Society of Soil Science 28(4): 733-735.

Subbiah, B. V. and Asija, G. L. 1956. A rapid procedure for the estimation of available nitrogen in soils. Current Science 25: 259.

Takkar, P. N. and Mann, M. S. 1975. Evaluation of analytical methods of estimation of available $\mathrm{Zn}$ in major soil series of Ludhiana, Punjab. Agrochimica 19: 420-430.

Takkar, P. N. and Randhawa, N. S. 1978. Micronutrients in Indian Agriculture. Fertilizer News 28(8): 3-26.

Talib, A. R. 1986. Soil and water conservation measures to combat Desertification in Cold Arid region of the Himalayas National seminar on desert Development at Leh. 23-25 Sep.

Tandon, H. L. S. 1989. Secondary and micronutrient recommendations for soils and crops-A Guide Book. FD and consultation organization C 110, Greater Kailash, 1, New Delhi.

Walkley, A. and Black, I. A. 1934. An examination of the Degtjareff method for determining soil organic matter and proposed modification of the chromic acid titration method. Soil Science 37: 29-38.

Wani, G. N. 2001. Studies on the distribution of secondary nutrient elements $(\mathrm{Ca}, \mathrm{Mg}$ and $\mathrm{S}$ ) in apple orchard soils of north Kashmir. M.Sc., thesis submitted to Sher-e-Kashmir University of Agricultural Science \& Technology of Kashmir pp. 75.

Zaho, J., Yan, D., Xingsbin, X., Xin, L., Xianxian, Z. and Xiang, S. 2011. Effect of annual variation in soil $\mathrm{pH}$ on available soil nutrients in pear orchards. Acta Ecologica Sinica 31: 212-216.

Zahoor, A., Tahir, A., Shahid, A. and Kirmani, N. A. 2005. Correlation studies on micro-nutrient cations with soil properties of apple orchard of Kashmir. Indian Journal of Horticulture 62(4): 391-393.

\section{How to cite this article:}

Mansoor Ali, M.A. Malik, Tsering Dolkar and Ahmad Ali. 2018. Nutrient Status of Aprioct (Prunus armeniaca L.) Orchards of District Kargil, Ladakh, India. Int.J.Curr.Microbiol.App.Sci. 7(06): 257-277. doi: https://doi.org/10.20546/ijcmas.2018.706.031 